\title{
Neuropsychiatric Complications of Efavirenz Therapy: Suggestions for a New Research Paradigm
}

\author{
Stefan Sütterlin, Dipl.-Psych. \\ Claus Vögele, Ph.D. \\ Siegfried Gauggel, Ph.D.
}

This review gives an up-to-date account of the current state of research on neuropsychiatric complications associated with efavirenz therapy and critiques the methods used in previous studies. The authors suggest an extension of current research strategies using psychophysiological concepts and including behavioral regulation models. From a practical perspective, these may contribute to better screening methods for the identification of those at risk for neuropsychiatric complications and the improvement of neuropsychiatric monitoring during efavirenz treatment. From a theoretical viewpoint, the suggested research paradigms may help to move beyond the current state of descriptive approaches and thus improve our limited understanding of underlying mechanisms.

(The Journal of Neuropsychiatry and Clinical Neurosciences 2010; 22:361-369)
$\mathrm{E}$ favirenz is a commonly used non-nucleoside re- verse transcriptase inhibitor frequently prescribed in combination with other antiretroviral regimens (e.g., protease inhibitors) for the treatment of HIV. After its admission for pharmaceutical treatment in the United States in 1998, efavirenz has become a cornerstone for highly active antiretroviral therapies (HAART). Good CNS penetration has been seen as a major strength of antiretroviral regimens, particularly efavirenz. Accumulating evidence suggests neuroprotective effects against cognitive decline such as HIV-associated dementia and other aspects of neuropsychological functioning. ${ }^{1-4}$ Nevertheless, its proven effectiveness as a first-line treatment in suppressing plasma viral load comes along with reports of adverse central-nervous and neuropsychiatric effects which have been frequently reported after exposure to efavirenz. ${ }^{5-9}$

Types and Prevalence Rates of Efavirenz-Associated Neuropsychiatric Effects: Current Evidence Depression, suicidal ideation, aggressive/impulsive behavior, paranoid reactions, manic reactions and (largely

Received May 19, 2009; revised August 13 and September 30, 2009; accepted October 5, 2009. Mr. Sütterlin and Dr. Vögele are affiliated with the Research Unit INSIDE at the University of Luxembourg; Dr. Gauggel is affiliated with the Institute of Medical Psychology and Medical Sociology at RWTH Aachen University, in Aachen, North Rhine-Westphalia, Germany. Address correspondence to Stefan Sütterlin, Dipl.-Psych., University of Luxembourg, Research Unit INSIDE, Building XII, Room 201C, Route de Diekirch, L-7220 Walferdange, Luxembourg; Stefan.Suetterlin@uni.lu (e-mail).

Copyright (c) 2010 American Psychiatric Publishing, Inc. 
anecdotal reports of) psychosis-like behavior, suicide, and severe delusions are among the most frequently reported psychiatric symptoms associated with efavirenz treatment. Dizziness, insomnia, impaired concentration, irritability, nervousness, somnolence, abnormal dreams, and hallucinations are usually classified as CNS symptoms. The differentiation between CNSrelated and psychiatric symptoms is rather arbitrary and has not been consistent; in general, psychiatric symptoms include some sort of behavioral or conscious component. This differentiation aims to reflect differences at the symptom level but does not necessarily imply distinct causal mechanisms.

Estimates of prevalence rates have varied over the years in line with the progress of efavirenz toxicity research, from early anecdotal case reports ${ }^{10-15}$ and cross-sectional controlled trials ${ }^{16-18}$ to prospective studies of larger samples ${ }^{19}$ and a growing number of reviews. ${ }^{5-7,9}$ Recent studies have focused on the relevance of plasma concentrations and genetic predispositions as moderators of neuropsychiatric complications. ${ }^{20-25}$

The Food and Drug Administration ${ }^{8}$ reported results from a sample of 1,008 patients with HIV who received treatment with efavirenz, and it stated the probability of "selected nervous-system symptoms of any severity" regardless of causality as $52.7 \%$ compared with $24.6 \%$ in a control group (HIV + individuals not receiving efavirenz). The risk of discontinuation of treatment as a result of experiencing symptoms was $2.1 \%$ in efavirenztreated patients compared with $1.1 \%$ in the control group. On average, prevalence rates range from $40 \%$ to $70 \% .{ }^{15,26-28}$ This wide range in prevalence estimates is probably due to differences in study design (e.g., sample sizes, recruitment methods, and assessment of neuropsychiatric complications) and methodological quality, but it is also due to the inconsistent use of terms such as "CNS-related toxicity," "psychiatric," and "neuropsychiatric," and categorizing mental disorders such as major depression or posttraumatic stress disorder using unspecified diagnostic criteria. To complicate the picture further, few studies have used assessment tools that are suitable for detecting efavirenz treatmentassociated complications on a subclinical level. Taking into account such methodological differences in early publications on efavirenz toxicity, reports on prevalence rates have since converged and remain relatively stable; an average of one out of two patients receiving efavirenz shows adverse psychiatric reactions. Repeat- edly confirmed in numerous controlled trials, these data refer to the incremental contribution of risk, which cannot be explained by HIV-associated long-term brain damage. In direct comparison to treatment alternatives, adverse effects resulting from treatments including efavirenz are reported three times more often than in cases of protease inhibitors. ${ }^{7}$

In terms of symptom manifestation over time, the most severe toxicity effects of efavirenz treatment are consistently reported to occur within the first $2-4$ weeks after efavirenz initiation, ${ }^{7}$ and symptoms generally cease after 6-8 weeks., ${ }^{5,9}$ Long-term effects covering periods of more than 12 weeks after efavirenz treatment onset have rarely been investigated. Following a pragmatic approach, studies have usually focused on clinically relevant symptoms that could impact on medication adherence rather than the investigation of causal pathways of the reported adverse reactions. Consequently, most studies prioritized the application of assessment tools and research designs suitable for the diagnosis of mental disorders rather than the identification of subclinical symptoms.

Subsequent research has reported mild and clinically tolerable persistent impairments of psychological functioning for up to 2 years after treatment onset. ${ }^{16}$ The Efavirenz Consensus Working Group and other researchers ${ }^{29,30}$ published recommendations for the treatment of efavirenz-induced adverse effects, including psychopharmacological treatment as well as nonpharmacological symptom-specific management strategies such as psychotherapeutic treatment.

\section{Methodological Shortcomings in Previous Research}

Research on efavirenz treatment-associated neuropsychiatric toxicity suffers from a range of methodological shortcomings, which have rarely been addressed in previous reviews. First, studies have often neglected the assessment of a psychiatric baseline status, often due to their retrospective design. ${ }^{31}$ Occasionally, interviewees were asked to provide a retrospective report on their mental status before treatment. ${ }^{32}$ In neither of the published prospective studies was a psychiatric, psychological, or neuropsychological baseline status before efavirenz treatment onset statistically controlled for, although psychiatric history is considered a relevant predictor for neuropsychiatric effects triggered by efavirenz. Second, the few published prospective studies suffer from high attrition rates and are thus very likely to underestimate the prevalence of psychiatric disor- 
ders in the actual outcome of clinical trials. ${ }^{27}$ This underestimation is caused by selection biases and the likely association of poor adherence and psychiatric disorders at treatment onset, which is often not assessed. Third, assessments in the form of semistructured interviews were applied by either researchers not blinded to the treatment condition (e.g., prescribing physicians), those without suitable training in the conduct of the interview, or both. ${ }^{19}$ Fourth, in the vast majority of studies, nonstandardized assessment tools were used or measures applied that are insensitive for the detection of prodromal symptoms. ${ }^{16,31,32}$

While the almost exclusive use of self-report measures in previous studies has enabled the identification of clinically important symptoms, their lack of specificity in relation to neurophysiological pathways does not allow for any conclusions to be drawn about the mechanisms linking efavirenz and neuropsychiatric complications. In addition, assessment tools such as screening tests and symptom checklists appear to be too insensitive and too global to detect symptoms at the subclinical level. These assessments are, therefore, likely to result in type II error, particularly in monitoring more subtle long-term effects. Statistical power, effect sizes, or both were rarely reported, though this information would be crucial in order to adequately assess the appropriateness of the applied assessment procedures and the severity of adverse reactions in samples that range from single case studies to large cohorts.

A particularly striking omission from previous research investigating efavirenz treatment-associated side effects concerns the almost complete lack of appropriate neuropsychological assessments. The only published and frequently cited studies claiming to have explicitly investigated neuropsychological symptoms related to efavirenz treatment are those from Clifford et al. ${ }^{33}$ and von Giesen et al. ${ }^{31}$ Both studies conclude that neuropsychological symptoms did not occur or were only mild and temporary. Nevertheless, the selection of subtests from standardized assessment tools was not theoretically justified in either study. For example, Clifford et al. ${ }^{33}$ used composite scores ("NPZ3") that were inappropriately derived (adding subscores of Trail Making tests A and B to the total of its subscales), omitted crucial neuropsychological domains, and were unspecific. These scores, therefore, do not allow for a comprehensive assessment of the "neuropsychological status" of patients with HIV. Furthermore, the previously reported ${ }^{34-37}$ observation that antiretro- viral therapy leads to early improvements in motorrelated tasks impairs the interpretational power of the results in terms of neuropsychological functioning.

von Giesen et al. ${ }^{31}$ used the "Kurztest zur Erfassung von Gedächtnis- und Aufmerksamkeitsleistungen" (Short Test for the Assessment of Memory and Attention Performance). This is a screening tool designed to detect early dementia without sufficient differentiation of memory and attention. It is, thus, insensitive to subtle or moderate changes in cognitive function related to efavirenz treatment, especially when compared to HIV patients not receiving efavirenz but likely to show HIVrelated impairments. In addition, assessors were not blinded to the treatment condition. The study is, therefore, methodically flawed, and its results (91\% of participants scored 0, "no severe cognitive impairment") are probably due to a floor effect. The authors' conclusion that "the present data do not provide evidence for a higher incidence of light and/or severe neuropsychiatric side effects" (p. 382) associated with efavirenz treatment is, therefore, questionable and unsupported by their evidence.

In summary, no extensive or theory-driven neuropsychological assessment has been attempted in studies on efavirenz toxicity. Current conclusions regarding neuropsychological performance have to be considered premature and largely unsupported by evidence.

Following a bottom-up approach, recent research has investigated the influence of the G516T polymorphism at the CYP2B6 isoenzyme on intracellular efavirenz plasma levels and has identified a CYP2B6 genotype as a predictor of efavirenz neuropsychological toxicity. ${ }^{23,25}$ Patients with a homozygotic 516G $>$ T substitution show higher efavirenz plasma levels and report more frequent adverse reactions to efavirenz treatment due to less effective intracellular drug clearance compared with those carrying the wild type (GG). These results could not be replicated in one other study. ${ }^{22}$

Nevertheless, the findings from Lowenhaupt et al. ${ }^{23}$ and Rotger et al. ${ }^{25}$ point to the possibility that previous investigations of efavirenz-related adverse effects may have suffered from low statistical power as they did not take into account inter-individual differences in drug clearance capacity. Drug clearance or efavirenz plasma levels are obvious moderators of any outcome in studies investigating toxicity associated with efavirenz treatment. Future research investigating causal pathways between 
efavirenz treatment and its adverse side effects should, therefore, screen participants for carriers of the G516T allele or include a measure of efavirenz clearance, or both, in order to identify those subsamples with increased vulnerability because of slow drug clearance.

\section{Questions Unanswered by Current Research}

Efavirenz is only one of several regimens in the class of retroviral inhibitors. Nevertheless, its extraordinary effective penetration into the CNS and its unique approach to the inhibition of viral reverse transcriptase have earned efavirenz the status of a first-line treatment despite its significantly higher risk of neurologic toxicity compared with other regimens of antiretroviral therapy. The reasons why neuropsychiatric effects occur more frequently and with greater severity during efavirenz treatment than, for example, during treatment with the structurally comparable nevirapine ${ }^{6,7}$ remain unclear, as the identification of neural pathways and systems responsible for these adverse effects is still pending.

Early case studies from the late 1990s and conference contributions on toxicity related to efavirenz treatment ${ }^{38}$ triggered a fast-growing pool of empirical data feeding into early reviews on efavirenz toxicity, ${ }^{15}$ recommending the monitoring of CNS adverse effects in all patients taking efavirenz. Psychiatric diagnoses, however, often remained rather vague, with some publications citing merely anecdotal observations without using standardized assessment methods or specified diagnostic criteria. There was a preponderance of pragmatic approaches in terms of risk management and the development of practice guidelines. Research designs and their implications were, therefore, rarely discussed. This practice-oriented "bottom-up approach" can be contrasted with a "top-down approach," which links the results on efavirenz treatment-associated symptoms with theoretical models from the behavioral sciences. Such an approach may contribute to a more comprehensive understanding of the complex interplay of cellular level mechanisms and behavioral patterns.

We argue that a shift in focus from clinical treatment-related toxicities to the development of a conceptual framework would allow for the improvement of screening methods for individuals at risk for adverse reactions following efavirenz treatment. This paradigm shift would also help to improve monitoring methods (early alerts) and contribute to the development of tests applied to detect neuropsychiatric risk potential for future drugs taking effect in the CNS.

\section{Suggestion for a New Research Approach}

Previous research on efavirenz treatment-associated neuropsychiatric toxicity has been largely descriptive, with the aim to identify, classify, and assess the probability and severity of symptoms that may impair treatment adherence for this effective and daily administered lifelong medication. Despite considerable improvements in risk management by including genotyping in medical decisions in vulnerable populations and recommendations for risk management strategies, the missing link between pathological symptoms and efavirenz has not been identified yet. Current research, therefore, is confined to providing practice guidelines for clinicians to maximize adherence rather than exploring their origin (e.g., alternative treatment options, dose reduction, prescription of antidepressants, and nonpharmacological interventions).

We argue, therefore, for the development of a theorydriven and standardized way of screening for neuropsychiatric toxicities in efavirenz and other antiretroviral agents. We suggest a top-down approach by classifying and integrating typical efavirenz-related symptoms such as depression, suicidal ideation, aggressive/impulsive behavior, delusions, impaired concentration, irritability, and nervousness into the psychological concept of self-regulation. This approach complements existing bottom-up research strategies in medical research and makes use of the profile of clinical symptomatology associated with efavirenz treatment, indicating an impairment of functions which can largely be conceptualized as behavioral, cognitive and affective dysregulation (Figure 1).

Concepts and methods from psychophysiology and experimental and clinical neuropsychology can locate current results in a theoretical framework, which will generate testable hypotheses that go beyond the selection of appropriate assessment tools. Such a model would allow for the identification of subtle effects of efavirenz on psychophysiological levels, leading to the identification of the neuronal networks affected. The similarities of efavirenzassociated symptoms to syndromes observed in braindamaged patients with dysexecutive functions and their involvement of systems of behavioral control suggest the application of standard paradigms designed for neuropsychological profiling in patients with inhibition-related impairments. ${ }^{39}$ Results from the neurosciences and psy- 


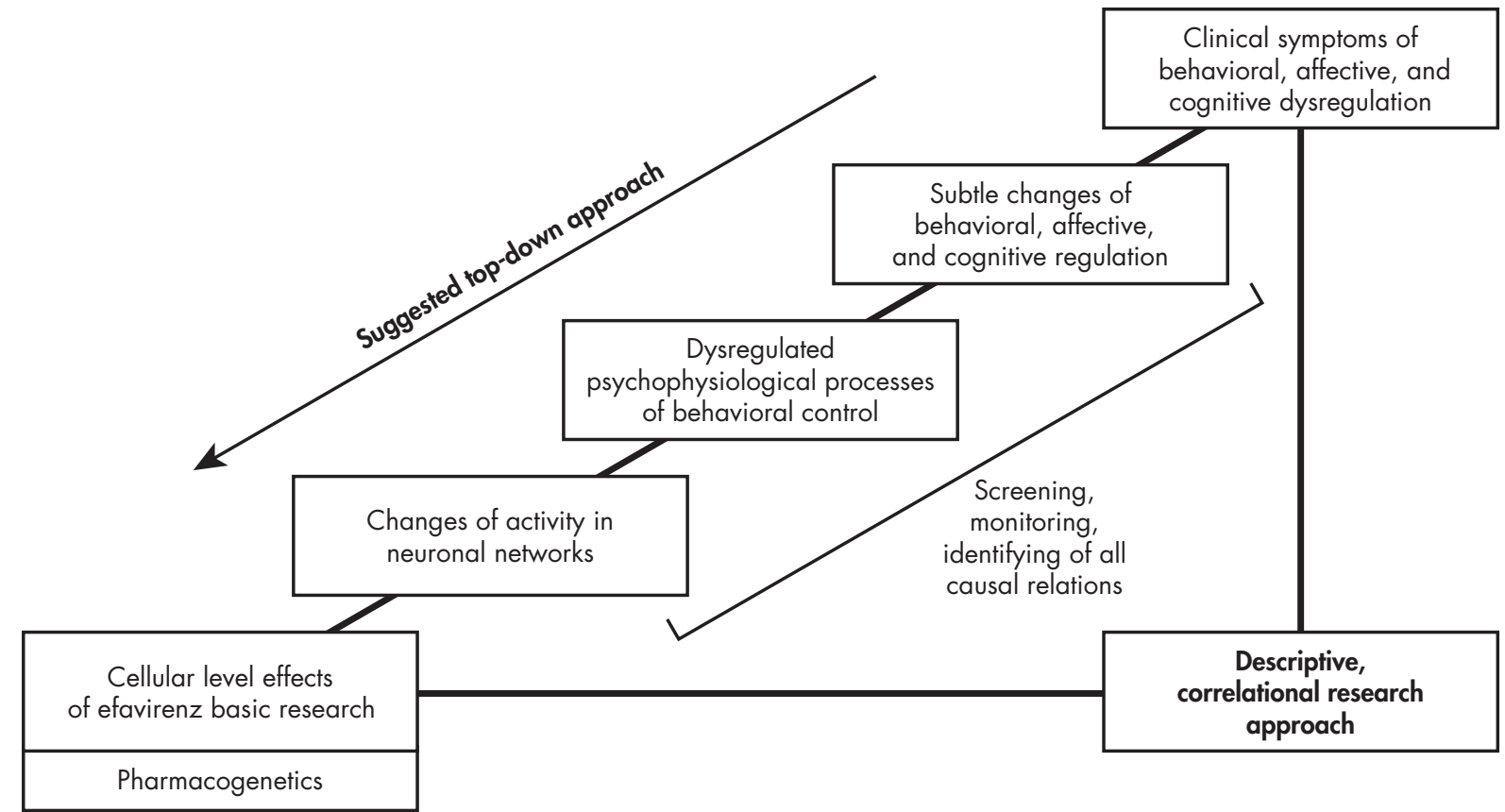

This theory-driven approach applies psychological models of behavioral regulation to observed manifestations of efavirenz treatmentrelated toxicities. Psychophysiological concepts link the subjective and social perspective of human behavior and experience with physiological correlates, which can be systematically assessed using standardized techniques. Empirical evidence for neuropsychological underpinnings of psychophysiological phenomena provides theoretical connections to neuronal activity patterns resulting in maladaptive behavior. A comprehensive approach making use of psychophysiological heuristics offers standardized and practical tools which might be applied in screening, monitoring, and fundamental research.

chology have established the capacity to self-regulate behavior, to resist impulses, and to regulate emotions effectively as a personality trait. ${ }^{40}$ Behavioral neuroscience of self-regulation links traditional psychological approaches of behavioral theory with their neurological underpinnings and implies standardized assessment tools for finegrained analyses of cognitive and behavioral regulative skills and their neuronal substrates, basically located in the frontal cortex. ${ }^{41}$ Behavioral self-regulation is often understood as one of several functions within the broad and sometimes fuzzy concept of executive functions. Research on dysexecutive syndromes provides models and specific experimental paradigms ${ }^{39}$ further supported by neuroimaging techniques which have not been used in efavirenzrelated research on psychiatric or psychological adverse effects. ${ }^{42,43}$ Neuropsychological and psychophysiological research on self-regulation makes use of assessment techniques that detect and quantify subtle neuronal and physiological processes relevant for behavioral control such as frontal inhibitory functions.

To give a more specific example for potentially interesting new research paradigms linking symptom obser- vations with established psychophysiological research paradigms, the assessment of peripheral parameters such as cardiac autonomic balance might contribute to a better understanding of behavioral dysregulation in efavirenz-treated patients. The neuroanatomical concept of central autonomic networks ${ }^{44,45}$ depicts a process of neurovisceral integration in which peripheral psychophysiological reactions (heart rate, salivation, electrodermal reactions, and startle reflex potentiation) are related to neocortical structures such as the prefrontal cortex (PFC) and cingulate cortex as well as subcortical limbic areas (thalamus, hypothalamus, and amygdalae), triggering autonomic reactions. The degree of flexibility of this system is related to adaptive behavior, including complex behavioral processes such as social interaction. On a peripheral level, this is expressed by vagal tone. Reciprocal cortico-cardiac interactions are key features of the central autonomic networks and are indicated by the sympathetic-parasympathetic interplay as it is expressed in heart rate variability. Autonomic nervous system-related measures such as heart rate variability or baroreceptor sensitivity can be con- 
ceived as a proxy for sympatho-vagal-balance, and due to their neuroanatomic relation to the PFC, they also indicate an individual's self-regulative capacities. ${ }^{46,47}$

Observed deficits in behavioral regulation as described in cases of efavirenz-induced neuropsychiatric reactions fit well into the psychological concepts of selfregulation, which cognitive neuroscience interprets as behavioral correlates of executive functions associated with the PFC, the ventro-medial PFC, and the cingulate cortex. These neuronal correlates and their corresponding autonomic functions, as predicted in central autonomic network theory, allow the deployment of established and standardized assessment tools. Hence, by applying new theory-driven research approaches, drug-induced behavioral abnormalities can be illuminated, screening methods improved, and treatment options considered.

The concept of central autonomic networks emphasizes the reciprocal interactions between (prefrontal) cortical and cardiac processes and is in line with frontal hypofunction in patients who show symptoms of behavioral, affective, or cognitive dysregulation. Making use of the reciprocity of the central-autonomic networks, an exemplary application of this concept could involve biofeedback of heart rate variability or respiratory sinus arrhythmia. This method is easy to use and has established efficacy in the treatment of depression ${ }^{48}$ and anxiety disorders. ${ }^{49}$ The reciprocity of cortico-cardiac interactions, based on cortical plasticity, provides opportunities for behavioral interventions. ${ }^{50,51}$

Electrophysiological correlates of behavior (e.g., event-related potentials) illuminate features of cognitive top-down and bottom-up processes such as evaluation, categorization, orienting/alertness, or novelty and are sensitive to pharmacological treatments. Several features such as amplitude and latency of eventrelated potentials can be linked to behavioral or cognitive dysregulation. Response inhibition paradigms combine behavioral tasks requiring motor inhibition in standardized and well-established paradigms such as Go-NoGo, ${ }^{48,49}$ stop-signal, ${ }^{53}$ or others with corresponding electrophysiological measures such as N200 amplitude. ${ }^{54} \mathrm{~A}$ sensitive measure such as the inhibition-related N200 event-related potential may be able to assess subclinical changes in cognitive and behavioral regulation processes, ${ }^{55}$ which have been suggested to be based on a supermodal inhibition-eliciting initiatior in the right inferior prefrontal cortex and, therefore, to be closely linked to cognitive adaptivity and flexibility. ${ }^{56,57}$
Symptomatic manifestations of dysregulated behavior typically following efavirenz toxicity suggest this method's relevance for possibly related symptoms for pharmacological treatment. The neural generators, which compose the N200 and the relevance of these anatomical structures for behavioral and cognitive regulation, are well researched. The same applies to other eventrelated potentials occurring early or late over the time course. They indicate features of cognitive processing and thus provide a quantitative assessment with much higher temporal resolution than through solely behavioral observation, though their selection is based on self-report information.

The methods outlined above are examples of standardized tools that assess behavioral and emotional regulation. They have been shown to contribute to a better understanding of a variety of affective and behavioral disorders covering the same class of symptoms of disinhibition as described in the literature on efavirenz side effects (e.g., impulsivity, ${ }^{58}$ affective disorders, ${ }^{59,60}$ psychopathology in general ${ }^{44,61}$ and various other psychiatric symptoms, impairments of executive functions, 39,42,43,59 and cognitive performance ${ }^{62}$ ) (Table 1 ).

Models of and empirical findings on executive functions and their relation to frontal inhibition and behavioral regulation ${ }^{42}$ can provide an extensive framework for behavioral regulation. Such concepts link neuroanatomical structures and psychophysiological processes with observable behavior in everyday life $^{63,64}$ and enable researchers to a theory-driven choice of specific and sensitive assessment methods.

We hope this new research approach will help to improve guidelines for efavirenz treatment by providing more sensitive assessment tools (i.e., those that are able to detect subtle subclinical changes, thus enabling practitioners to prevent or minimize treatment-associated adverse effects). We further expect progress in research on the identification of persons at risk for adverse effects of efavirenz treatment and argue for the introduction of paradigms developed in behavioral sciences to be applied in research on drug side effects with psychological and psychiatric implications.

\section{CONCLUSION}

In this review we suggest a change in research paradigms and conceptualize efavirenz-related side effects as manifestations of behavioral dysregulation. We pro- 
TABLE 1. Suggested Methodological Approaches for the Identification of Sub-Clinical Toxic Effects

\begin{tabular}{lcc}
\hline Toxic Effects & Data Level & Exemplary Assessment Methods \\
\hline $\begin{array}{c}\text { Subtle changes of behavioral, affective } \\
\text { and cognitive regulation }\end{array}$ & $\begin{array}{c}\text { Self-report of sub-clinical symptoms } \\
\text { in everyday context }\end{array}$ & $\begin{array}{c}\text { Standardized questionnaires for the assessment } \\
\text { of prodromal symptoms in non-clinical } \\
\text { populations: e.g., CES-D, Barret Impulsivity }\end{array}$ \\
$\begin{array}{l}\text { Scale, Behavior Rating Inventory of } \\
\text { Executive Function (BRIEF), etc. }\end{array}$ \\
$\begin{array}{c}\text { Dysregulated psychophysiological } \\
\text { processes of behavioral control }\end{array}$ & $\begin{array}{c}\text { Response inhibition; Heart rate variability; } \\
\text { electrodermal activity; distractibility and } \\
\text { attentional biases }\end{array}$ \\
$\begin{array}{c}\text { Changes of activity patterns in } \\
\text { neuronal networks }\end{array}$ & $\begin{array}{c}\text { autonomic function } \\
\text { Event-related potentials (N100, N200, P3a/b, } \\
\text { etc.); frontal alpha-asymmetries }\end{array}$
\end{tabular}

The suggested methodological approaches and assessment tools are only exemplary and can be extended. CES-D=Center for Epidemiologic Studies Depression Scale

pose to extend current research strategies to a theorydriven top-down process tying together existing knowledge on self-regulation theory. Previous research on efavirenz toxicity is restricted to purely descriptive approaches at the symptom level. By suggesting a topdown, theory-driven strategy we argue for applying established and standardized assessment tools, which are provided by the psychophysiological concepts of self-regulation. The aims of this reconceptualization are the improvement of screening methods for the identification of patients at risk for efavirenz treatment-related side effects, the enhancement of fundamental research on self-regulation-related effects of pharmacological substances passing the blood-brain barrier, and the establishment of effective and close monitoring during treatment. This approach enables researchers to tap into established knowledge in order to choose specific and sensitive psychophysiological assessment tools based on hypotheses derived from observation and self-reports of previous research. The use of psychophysiological measures in the assessment process enables the quantification of treatment-related side effects with high temporal solution, comparative measurements, and sensitivity toward subclinical effects, and thus provides a new source of information.
The assessment methods suggested here are established and standardized; data for various common psychological and psychiatric disorders frequently described as efavirenz-related adverse effects are available. This will generate hypotheses for risk assessment and risk management, screening methods, monitoring, and prescription criteria and opens new perspectives for the investigation of neuronal networks and pathways. We argue that the identification and quantification of efavirenz treatment-related subtle and subclinical changes on cognitive, behavioral, and emotional levels are crucial for substantial progress from purely descriptive approaches of the past toward an understanding of psychopharmacological effects. These subtle changes require appropriate research designs (controlled, double-blinded) and standardized assessment tools beyond behavioral observation and self-report in order to tap into existing knowledge of behavioral regulation and its origins. This approach and its related methodology address serious limitations in current research by bridging the gap between fundamental research in neurobiological domains and behavioral sciences.

This paper has benefitted in its early stages from discussions with Dr. Rupert Whitaker, Tuke Institute, London.

\section{References}

1. Cysique LA, Vaida F, Letendre S, et al: Dynamics of cognitive change in impaired HIV-positive patients initiating antiretroviral therapy. Neurology 2009; 73:342-348

2. Liner KJ, Hall CD, Robertson KR: Effects of antiretroviral therapy on cognitive impairment. Curr HIV / AIDS Reports 2008; 5:64-71

3. Pollicita M, Aquaro S, Svicher V, et al: HIV-1-associated dementia during HAART therapy. Recent Pat CNS Drug Discov $2008 ; 3: 23-33$
4. Wynn HE, Brundage RC, Fletcher CV: Clinical implications of CNS penetration of antiretroviral drugs. CNS Drugs 2002; 16:595-609

5. Arendt G, de Nocker D, Giesen HJ, et al: Neuropsychiatric side effects of efavirenz therapy. Expert Opin Drug Saf 2007; 6:147-154

6. Barreiro P, García-Benayas T, Soriano V, et al: Simplification of antiretroviral treatment: how to sustain success, reduce toxicity and ensure adherence avoiding PI use. AIDS 2002; 4:233-241 
7. Cespedes MS, Aberg JA: Neuropsychiatric complications of antiretroviral therapy. Drug Saf 2006; 29:865-874

8. World Health Organization: Efavirenz 200 mg Capsules (Ranbaxy), HA305. 2007. Available at http://apps.who.int/prequal/ WHOPAR/WHOPARPRODUCTS/HA305Part6v1.pdf

9. Treisman GJ, Kaplin AI: Neurological and psychiatric complications of antiretroviral agents. AIDS 2002; 16:1201-1215

10. Allavena C, Le Moal G, Michau C, et al: Neuropsychiatric adverse events after switching from an antiretroviral regimen containing efavirenz without tenofovir to an efavirenz regimen containing tenofovir: a report of nine cases. Antivir Ther 2006; 11:263-265

11. Foster R, Olajide D, Everall IP: Antiretroviral therapy-induced psychosis: case report and brief review of the literature. HIV Med 2003; 4:139-144

12. de la Garza CL, Paoletti-Duarte S, García-Martín C, et al: Efavrienz-induced psychosis. AIDS 2001; 15:1911-1912

13. Moreno A, Labelle C, Samet JH: Recurrence of post-traumatic stress disorder symptoms after initiation of antiretrovirals including efavirenz: a report of two cases. HIV Med 2003; 4:302304

14. Peyriere H, Mauboussin JM, Rouanet I, et al: Management of sudden psychiatric disorders related to efavirenz. AIDS 2001; 15:1323-1324

15. Puzantian T: Central nervous system adverse effects with efavirenz: case report and review. Pharmacotherapy 2002; 22: 930-933

16. Fumaz CR, Muñoz-Moreno JA, Moltó J, et al: Long-term neuropsychiatric disorders on Efavirenz-based approaches. Clin Sci 2005; 38:560-565

17. Hawkins T, Geist C, Young B, et al: Comparison of neuropsychiatric side effects in an observational cohort of efavirenzand protease inhibitor-treated patients. HIV Clin Trials 2005; 6:187-196

18. Rihs TA, Begley K, Smith DE, et al: Efavirenz and chronic neuropsychiatric symptoms: a cross-sectional case control study. HIV Med 2006; 7:544-548

19. Pérez-Molina JA: Safety and tolerance of efavirenz in different antiretroviral regimens: results from a national multicenter prospective study in 1,033 HIV-infected patients. HIV Clin Trials 2002; 3:279-286

20. Haas DW, Ribaudo HJ, Kim RB, et al: Pharmacogenetics of efavirenz and central nervous system side effects: an adult AIDS clinical trials group study. AIDS 2004; 18:2391-2400

21. Hasse B, Günthard HF, Bleiber G, et al: Efavirenz intoxication due to slow hepatic metabolism. Clin Infect Dis 2005; 40:22-23

22. Kappelhoff BS, Leth F, Robinson PA, et al: Are adverse events of nevirapine and efavirenz related to plasma concentrations? Antivir Ther 2005; 10:489-498

23. Lowenhaupt EA, Matson K, Qureishi B, et al: Psychosis in a 12-year-old HIV-positive girl with an increased serum concentration of efavirenz. Clin Infect Dis 2007; 45:128-130

24. Marzolini C, Telenti A, Decosterd LA, et al: Efavirenz plasma levels can predict treatment failure and central nervous system side effects in HIV-1-infected patients. AIDS 2001; 15: 71-75

25. Rotger M, Colombo S, Furrer H, et al: Influence of CYP2B6 polymorphism on plasma and intracellular concentrations and toxicity of efavirenz and nevirapine in HIV-infected patients. Pharmacogenet Genomics 2005; 15:1-5
26. US Department of Health and Human Services: AIDSinfo. Available at http:/ / www.aidsinfo.nih.gov/

27. Blanch J, Martínez E, Rousaud A, et al: Preliminary data of a prospective study on neuropsychiatric side effects after initiation of efavirenz. J Acquir Immune Defic Syndr 2001; 27:336343

28. Goldenberg D, Boyle B: Psychiatric safety of efavirenz (abstract WePeB4238). Durban, South Africa, XIII Conference on AIDS, 2000. Available at http://gateway.nlm.nih.gov/ MeetingAbstracts $/ \mathrm{ma} \mathrm{f}=102240450 . \mathrm{html}$

29. Gill MJ, Rachlis A, Walmsley S, et al: Canadian Expert Panel recommendations on the management of CNS symptoms related to efavirenz. Can J Infect Dis 2001; 12:20c-30c

30. Halman M: Management of depression associated with HIV/ AIDS and antiretroviral therapy. Can J Infect Dis Med Microbiol 2006; 17:5D-9D

31. von Giesen HJ, Köller H, de Nocker D, et al: Long-term safety and efficacy of NNRTI within the central nervous system. HIV Clin Trials 2003; 4:382-390

32. Lochet $P$, Peyrière $H$, Lotthé $A$, et al: Long-term assessment of neuropsychiatric adverse reactions associated with efavirenz. HIV Med 2003; 4:62-66

33. Clifford DB, Evans S, Yang Y, et al: Impact of efavirenz on neuropsychological performance and symptoms in HIV-infected individuals. Ann Intern Med 2005; 143:714-721

34. Ferrando SJ, Gorp W, McElhiney M, et al: Highly active antiretroviral treatment (HAART) in HIV infection: benefits for neuropsychological function. AIDS 1998; 12:F65-F70

35. Ferrando SJ, Rablik JG, Gorp W, et al: Longitudinal improvement in psychomotor processing speed is associated with potent combination antiretroviral therapy in HIV-1 infection. J Neuropsychiatry Clin Neurosci 2003; 15:208-214

36. Giesen HJ, Theisen A, Arendt G: Therapeutic effects of nonnucleoside reverse transcriptase inhibitors on the central nervous system in HIV-1-infected patients. J Acquir Immune Defic Syndr 2002; 29:363-367

37. Sacktor N, Schifitto G, McDermott MP, et al: Transdermal selegiline in HIV-associated cognitive impairment: pilot, placebo-controlled study. Neurology 2000; 54:233-235

38. Ruiz NM, Bessen LJ, Manion DJ: Potential adverse experiences associated with efavirenz in adults (abstract 655). Chicago, 6th Conference on Retroviruses and Opportunistic Infections, 1999. Available at http://gateway.nlm.nih.gov/MeetingAbstracts/ ma?f=102195420.html

39. Stuss DT, Alexander MP: Is there a dysexecutive syndrome? Philos Trans R Soc Lond B Biol Sci 2007; 362:901-915

40. Baumeister RF, Vohs KD (eds): Handbook of Self-Regulation: Research, Theory, and Applications. New York, Guilford, 2004

41. Banfield JF, Wyland CL, Macrae CN, et al: The cognitive neuroscience of self-regulation, in Handbook of Self-Regulation. Edited by Baumeister RF, Vohs KD. New York, Guilford, 2004, pp 62-83

42. Fassbender C, Murphy K, Foxe J, et al: A topography of executive functions revealed by functional magnetic resonance imaging. Cogn Brain Res 2004; 20:132-143

43. Salmon E, Collette F: Functional imaging of executive functions. Acta Neurol Belg 2005; 105:187-196

44. Thayer JF, Brosschot JF: Psychosomatics and psychopathology: looking up and down from the brain. Psychoneuroendocrinology 2005; 30:1050-1058 
45. Thayer JF, Lane RD: A model of neurovisceral integration in emotion regulation and dysregulation. J Affect Disord 2000; 61:201-216

46. Knoch D, Fehr E: Resisting the power of temptations: the right prefrontal cortex and self-control. Ann N Y Acad Sci 2007; 1104:123-134

47. Segerstrom SC, Solberg Nes L: Heart rate variability reflects self-regulatory strength, effort, and fatigue. Psychol Sci 2007; 18:275-281

48. Siepmann M, Aykac V, Unterdörfer J, et al: A pilot study on the effects of heart rate variability biofeedback in patients with depression and in healthy subjects. Appl Psychophysiol Biofeedback 2008; 33:195-201

49. Reiner R: Integrating a portable biofeedback device into clinical practice for patients with anxiety disorders: results of a pilot study. Appl Psychophysiol Biofeedback 2008; 33:55-61

50. Colcombe SJ, Kramer AF, Erickson KI, et al: Cardiovascular fitness, cortical plasticity, and aging. Proc Natl Acad Sci U S A 2004; 101:3316-3321

51. Hansen AL, Johnsen BH, Sollers JJ, et al: Heart rate variability and its relation to prefrontal cognitive function: the effects of training and detraining. Eur J Applied Physiology 2004; 93: 263-272

52. Kawashima $\mathrm{R}$, Sato $\mathrm{K}$, Itoh $\mathrm{H}$, et al: Functional anatomy of GO/NO-GO discrimination and response selection: a PET study in man. Brain Res 1996; 728:79-89

53. Zheng D, Oka T, Bokura H, et al: The key locus of common response inhibition network for no-go and stop-signals. J Cogn Neurosci 2008; 20:1434-1442

54. Patel SH, Azzam PN: Characterization of N200 and P300: selected studies of the event-related potential. Int J Med Sci $2005 ; 2: 147-154$
55. Sumich AL, Kumari V, Heasman BC, et al: Abnormal asymmetry of N200 and P300 event-related potentials in subclinical depression. J Affect Disord 2006; 92:171-183

56. Konishi S, Nakajima K, Idai U, et al: Transient activation of inferior prefrontal cortex during cognitive set shifting. Nat Neurosci 1998; 1:80-84

57. Konishi S, Kyoichi N, Idai U, et al: Common inhibitory mechanism in human inferior prefrontal cortex revealed by eventrelated functional MRI. Brain 1999; 122:981-991

58. Chamberlain SR, Sahakian BJ: The neuropsychiatry of impulsivity. Curr Opin Psychiatry 2007; 20:255-261

59. Gorman JM, Sloan RP: Heart rate variability in depressive and anxiety disorders. Am Heart J 2000; 140:77-83

60. Friedman BH: An autonomic flexibility-neurovisceral integration model of anxiety and cardiac vagal tone. Biol Psychol 2007; 74:185-199

61. Thayer JF, Lane RD: Claude Bernard and the heart-brain connection: further elaboration of a model of neurovisceral integration. Neurosci Biobehav Rev 2009; 33:81-88

62. Thayer JF, Hansen AL, Saus-Rose E, et al: Heart rate variability, prefrontal neural function, and cognitive performance: the neurovisceral integration perspective on selfregulation, adaptation, and health. Ann Behav Med 2009; 37:141-153

63. Barkley R: The executive functions and self-regulation: an evolutionary neuropsychological perspective. Neuropsychological Rev 2001; 11:1-29

64. Miyake A, Emerson MJ, Friedman NP: Assessment of executive functions in clinical settings: problems and recommendations. Semin Speech Lang 2000; 21:169-183 